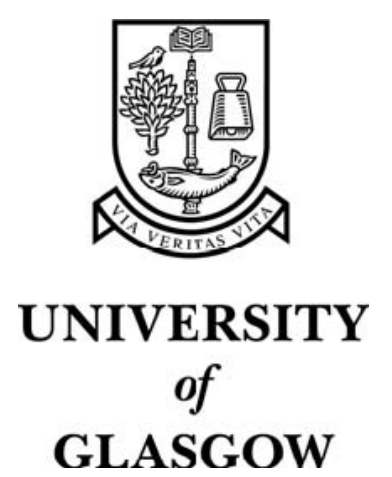

Al-Qassas, R. and Ould-Khaoua, M. and Mackenzie, L. (2006) Performance evaluation of a new end-to-end traffic-aware routing in MANETs. In, Proceedings of the 12th International Conference on Parallel and Distributed Systems - Volume 2 (ICPADS'06), 12-15 July 2006, pages pp. 49-54, Minneapolis, Minnesota, USA.

http://eprints.gla.ac.uk/3498/ 


\title{
Performance Evaluation of a New End-to-End Traffic-Aware Routing in MANETs
}

\author{
Raad S. Al-Qassas, Mohamed Ould-Khaoua, Lewis M. Mackenzie \\ Department of Computing Science \\ University of Glasgow \\ Glasgow, G12 8RZ, UK \\ Email: \{raad, mohamed, lewis\}@dcs.gla.ac.uk
}

\begin{abstract}
There has been a lot research effort on developing reactive routing algorithms for mobile ad hoc networks (MANETs) over the past few years. Most of these algorithms consider finding the shortest path from source to destination in building a route. However, this can lead to some network nodes being more overloaded than the others. In MANETs resources, such as node power and channel bandwidth are often at a premium and, therefore, it is important to optimise their use as much as possible. Consequently, a traffic-aware technique to distribute the load is very desirable in order to make good utilisation of nodes' resources. Therefore a number of end-to-end traffic aware techniques have recently been proposed for reactive routing protocols to deal with this challenging issue. In this paper we contribute to this research effort by proposing a new traffic aware technique that can overcome the limitations of the existing methods. Results from an extensive comparative evaluation show that the new technique has superior performance over similar existing end-to-end techniques in terms of the achieved throughput, end-to-end delay and routing overhead.
\end{abstract}

Keywords: Ad hoc networks, routing protocol, traffic, load balancing.

\section{Introduction}

A Mobile Ad hoc Network (MANET) is a collection of wireless mobile nodes that form a temporary network without the need for any infrastructure or centralized administration. In such an environment, it may be necessary for one mobile node to enlist the aid of others in forwarding a packet to its destination due to the limited propagation range of each mobile node's wireless transmissions [1]. The communication in MANETs is peer-to-peer as the mobile nodes communicate directly with one another. In MANET resources like power and bandwidth are at a premium and it is important to minimise the use of these resources.

The routing protocol in MANETs is responsible for establishing and maintaining paths between nodes in the network. The topology of a MANET may change frequently as nodes may move or power themselves off to save energy. In addition, new nodes can join the network [2].
Consequently, connectivity information is often required to be collected periodically in order to get a consistent view of the network, but this increases the bandwidth consumption resulting from collecting this information. MANETs have limited bandwidth, and therefore need an efficient routing protocol that can establish and maintain routes for both stable and dynamic topologies with minimum bandwidth consumption.

A major challenge in MANETs is the design of a routing protocol that can accommodate their dynamic nature and frequent topology changes; the topology can change unpredictably, so the routing protocol should be able to adapt automatically. However, when designing a protocol, it is not only the frequent changes in the network that are of concern, but also the natural limitations that these networks suffer from, such as limited bandwidth and power. To deal with such issues a number of routing protocols have been proposed [3, 4, 5, 6, 7, 8, 9, 10, 11, 12,13].

There has been a lot of work on developing reactive routing algorithms for ad hoc networks [3, 10, 12]. Most of these algorithms consider finding the shortest path from source to destination in building a route. However, this can lead to some nodes being overloaded more than others in the network. Therefore, a traffic-aware technique to distribute the load is highly desirable in order to make good utilisation of nodes' scarce resources. In addition it can be useful to prevent the creation of congested areas in the network, which can lead at the end into an improvement on the network performance. Furthermore, such a technique is a good way to achieve fairness in using node's limited resources.

A number of studies $[14,15,16,17,18]$ have recently proposed traffic-aware techniques for distributing the load in reactive routing. These techniques can be classified into two main categories: end-to-end and on-the-spot; based on the way they establish and maintain routes between any source and destination. The first category is based on using end-to-end information collected along the path from source to destination. In this category, intermediate nodes participate in building the route by adding some information about their status. However the decision for selecting the 
path is taken at one of the ends, either the source or the destination. In the second category, information is not required to be passed to one of the ends to make a path selection decision; it is most likely that an intermediate node will do this job. Therefore the decision of selecting a path is made on-the-spot and taken by intermediate nodes.

In this paper we present a new traffic aware routing technique that can overcome the limitations of the existing ones. A major limitation of existing techniques, such as those proposed in $[15,16]$, arise from the lack of information about the real traffic load experienced by nodes, which indeed affect the performance of the routing protocol and its efforts in distributing the load over nodes. A special characteristic of the new method over existing ones is that it takes more accurate information about traffic transiting a network node; this is computed by using the lengths of packets passed over nodes and the one waiting at the nodes' interface queue. The rationale behind using packets sizes in the calculations rather than just using the number of packets as in [15] is that packets can vary in size so it is better to use packets sizes as it can cover all the variations, and give a better indication of message contention. As a result, the new technique can potentially make a better judgment than the existing methods of $[15,16]$ in selecting routes, which improves the overall performance of the network, and distribute the load more fairly over the nodes in the network

The remainder of this paper is organised as follows. Section 2 reviews two existing end-to-end techniques, namely degree of nodal activity and traffic density. Section 3 describes the proposed traffic aware technique. Section 4 conducts a comparative study of the performance of the three techniques. Finally, section 5 concludes this study.

\section{End-to-end traffic aware techniques}

In this section we describe the two end-to-end techniques, degree of nodal activity proposed in Load-Balanced Ad hoc Routing (LBAR) [16] and traffic density defined in the routing algorithm Load Aware Routing in Ad hoc (LARA) [15].

\subsection{Degree of nodal activity}

The degree of nodal activity was defined in LBAR as a technique or metric for selecting the route with least traffic load. LBAR is a reactive routing protocol that focuses on how to find the path which would reflect the least traffic load based on a cost function. The cost function is calculated using two components: nodal activity and traffic interference. Nodal activity of a node is defined as the number of active paths passing through that node. An active path is an established path from a source to a destination. Traffic interference is defined as the sum of nodal activity for the node's immediate neighbours. The cost of a route is defined as the sum of a nodes' own nodal activity plus the activity of its neighbouring nodes. The path with minimum cost is that with minimum traffic and this is selected to be the route between source and destination.

Route discovery. The LBAR route discovery process is initiated whenever a source node needs to establish a path with another node. The source node broadcasts a setup message to its neighbours. The setup message carries the cost seen from the source to the current node. A node that receives a setup message will forward it to its neighbours after updating the cost based on its nodal activity value and traffic interference value. In order to prevent looping when setup messages are routed, the setup message contains a list of all node IDs used in establishing the path from source node to the current intermediate node. The destination node collects arriving setup messages within a route-select waiting period, which is a predefined timer for selecting the best-cost path. After the waiting period expires the destination sends an ACK message to the source node along the selected path. When the source node receives an ACK message, it recognises that a path has been established to the destination and then starts transmission.

Route maintenance. Route maintenance is triggered whenever a node on the active path moves out of the communication range, the case in which an alternate path must be found. If the source node moves away from the active path, the source has to reinitiate the route discovery procedure to establish a new route to the destination. When either the destination node or some intermediate node moves outside the active path, path maintenance will be initiated to correct the broken path. Once the next hop becomes unreachable, the node upstream of the broken hop propagates an error message to the destination node. The destination then picks up an alternative path and then sends an ACK message to the initiator of the error message. If the destination has no alternative path, it propagates an error message to the source, which will initiate a new route discovery if needed.

\subsection{Traffic density}

The traffic density was proposed in LARA as a metric for selecting the route with the minimum traffic load. LARA uses traffic density to represent the degree of contention at the medium access control layer. This metric is used to select the route with the minimum traffic load when the route is setup. The LARA protocol requires that each node maintain a record of the latest traffic queue estimations at each of its neighbours in a table called the neighbourhood table. Traffic queue is defined as the average value of the interface queue length measured over a period of time. Traffic density of a node is defined as the sum of the traffic queue of that node plus the traffic queues of all its neighbours. 
Route discovery. In LARA, the route discovery process is initiated whenever a node needs to establish a path with another node. In the route request process, the source broadcasts a route request packet that contains a sequence number, a source id and a destination id. A node that receives the request, broadcasts the request further, after appending its own traffic density to the packet. This process continues until the request packet reaches the destination. After receiving the first request, the destination waits for a fixed time-interval for more route request packets to arrive. When the timer expires, the destination node selects the best route from among the candidate routes and sends a route reply to the source. When the source node receives the route reply, it can start data transmission. If it does not receive any route reply within a route discovery period, it can restart the route discovery procedure afresh.

Route maintenance. Route maintenance is triggered whenever a node on the active path moves out of the communication range, in which case an alternate path must be found. If a link failure occurs during a data transmission session, the source is informed of the failure via a route error packet. On receiving a route error packet, the source initiates a new route request and queues all subsequent packets for that destination until a new route is found.

\section{The proposed load density technique}

The existing end-to-end traffic aware techniques use a metric or cost function to select the route with a minimum load, such techniques are represented by nodal activity [16] and traffic density [15]. The nodal activity metric cost function calculation is based on monitoring the number of active paths passed over nodes. On the other hand, the traffic density metric is measured using number of packets at interface queue. However in order to make a good judgment about a given path's load, it is not enough just to capture the number of active paths or number of packets at the interface queue over a period of time. Number of active paths can be useful when the used traffic flows are equal in characteristics. Number of packets at the interface queue is useful to capture the contention at the MAC layer if all packets are equal in size. However, this is not sufficient to represent the load. Therefore, what is needed is a metric that can deal with most of the cases that could appear in the network. Whether flows are with equal characteristics or not, or whether packets are equal in size or not, it should not affect the efficiency of the traffic aware technique. Our goal here is to devise a new end-to-end metric that selects the less congested route with the least traffic history regardless of the shape of the traffic passed over it.

Our proposed metric, named load density, is calculated using two main components; the load history information represented by the total traffic passed over nodes, and the contention information represented by and the number of packets waiting at the nodes' interface queue in order to take the possible contention in the network into consideration in the metric calculations. The load density is embedded under a reactive routing algorithm like other existing metrics the degree of nodal activity and traffic density. The sections below describe this algorithm. As an alternative solution to represent the contention, we can use the sum of packets' length occupying the queue as it can cover the variance in packets sizes instead of using the number of packets at interface queue.

Route discovery. The route discovery process starts whenever a node wants to communicate with another node for which it does not have a known route. The source node broadcasts a request packet to its neighbours. Every node receives the request packet will forward it to its neighbours after updating the cost information carried in the request packet, by adding the values of its load-history and contention information (see sec. 3.1) to those carried in the packet. The cost information carried in the request packet, which includes the load history and the contention information, represents the cost seen from the source to the current node. The process of forwarding the request packet continues until the packet is received by the destination node. The destination collects the arriving request packets within a route selection period; activated upon receiving the first request packet, for selecting the best-cost route. Once the selection period is expired the destination selects the route with the best cost and sends a reply packet to the source node. The route selection process is illustrated in more details in Fig. 1. When the source node receives the reply packet, the path is then established and communication can be started.

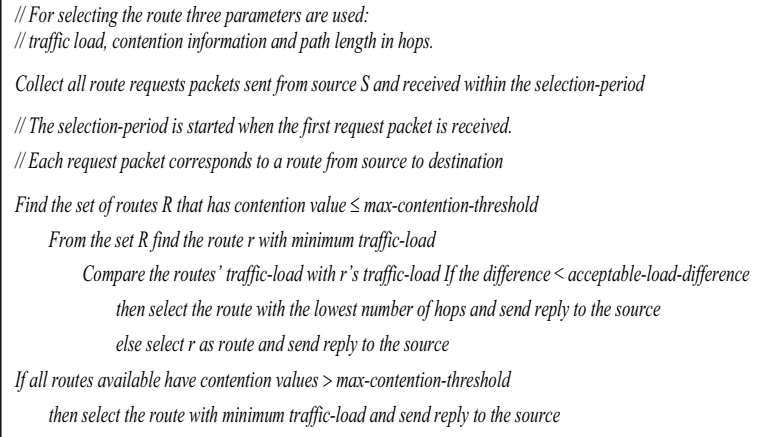

Fig. 1: Route selection algorithm in the new load aware technique.

Route maintenance. The route maintenance is triggered when there is a change in the topology that affects the validity of an active route. If the source node, an intermediate node or the destination node on an active route moves out of the communication range, an alternative route must be found. Once a node detects that the next hop is 
unreachable, it propagates an error message to the destination node. Upon receiving the error message, the destination node picks up an alternative route and then sends a reply message to the initiator of the error message. If the destination has no alternative path, it sends an error message to the source to start a route discovery process.

\subsection{Route cost computation}

The cost function has two main components: the data traffic load (in bytes) forwarded by nodes and number of packets at the interface queue. Every node keeps information about the amount of traffic passed over it during a predetermined period of time in addition to the interface queue history represented by the averaged number of packets occupying the queue over a period of time. Route cost is calculated by gathering traffic load and contention information for the nodes along the route. The contention information for a node represents the number of packets at the interface queue plus the number of packets at the interface queue for its neighbours.

Nodes exchange contention information using hello packets. Each node broadcasts a hello packets every hello interval, to its neighbours, containing its identity and contention information. The hello packet is broadcasted only for one hop i.e. only to the immediate neighbours. Neighbours who receive this packet update their neighbourhood information.

\section{Performance evaluation}

The performance merits of the existing end-to-end traffic aware techniques like traffic density [15] and nodal activity [16] have been analysed and compared against traditional routing algorithms [3, 12]. There has also been a performance comparison among the existing on-the-spot techniques in the study of [17]. However, there has not so far been a similar study that evaluates and compares the relative performance merits of end-to-end techniques. Therefore, one of our research goals is to undertake a thorough study of end-to-end techniques in situations where it is possible to select one from a set of feasible routes from source to destination in order to distribute the load where possible. This study will demonstrate advantages and disadvantages and applicability under various working environments. In our study the performance of the traffic density and nodal activity is assessed through simulations implemented using the well-known network simulator ns-2 [19].

\subsection{Simulation model}

The simulation model consists of the following main components: simulation area, simulation time, number of nodes, mobility model, maximum node speed, number of traffic flows, and traffic rate. The model is represented by two scenario files, which are the topology scenario and traffic scenario. The topology scenario corresponds to how nodes are distributed over the simulation area and their movement during simulation time. The traffic scenario file contains the type of data, number of flows, traffic rate, and flow start time and end time. In all scenarios nodes are assumed to be equipped with the wireless standard IEEE 802.11 with a transmission range of $250 \mathrm{~m}$ and a bandwidth of $2 \mathrm{Mbps}$.

In order to maximise the opportunity of forming multiple paths between data flow sources and their destinations we have chosen to assume that they are stationary while the rest of the nodes in the network are mobile. The reason for this is that sources could come within the range of each other or be very close to doing so due to mobility. Therefore keeping them stationary can boost our study of the traffic aware techniques. This, of course, will not create fixed paths between source and destination pairs as intermediate nodes that form the paths are mobile. Fig. 2 illustrates how sources and destination are placed in the topology.

We have implemented the traffic aware techniques, traffic density and nodal activity, under the AODV-like routing algorithm AOMDV [20]. AOMDV is a multi-path algorithm that supports loop-free multiple paths. The ns-2 source code for this algorithm is available and it is easier to modify this source code to simulate the traffic density and degree of nodal activity metrics rather than writing it from scratch.

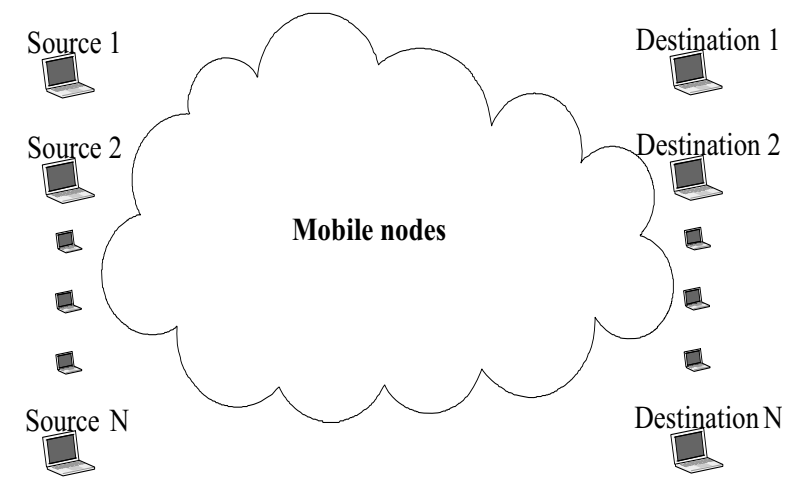

Fig. 2: Illustration of how sources and destinations are placed in the topology.

\subsection{Simulation Results}

The evaluation is based on the simulation of 100 wireless nodes forming a MANET over a flat space of size $1200 \mathrm{~m} \times$ $1000 \mathrm{~m}$ for a period of 900 seconds. Flows with Constant Bit Rate (CBR) data have been used. The traffic rate varied between 2, 4 and 8 packets per second representing low, medium and high traffic loads, respectively. The number of CBR flows is 5 flows with packet size of 512 bytes. Nodes move according to the widely used random waypoint model [3]. In the random waypoint model each node remains 
stationary for a pause time period. When the pause time expires, the node selects a random destination in the simulation space and moves towards it. When the node reaches its destination, it pauses again for the same pause time. This behaviour is repeated throughout the simulation time. In all the simulated scenarios the pause time has been set to 0 seconds to allow all time mobility. The node maximum speeds varied between $1,2,5,7,10,15$ and $20 \mathrm{~m} / \mathrm{s}$. For each speed we have made runs for 30 randomly generated topologies. Simulation parameters are illustrated in Table 1.

TABLE 1: The system parameters used in the simulation experiments

\begin{tabular}{|l|l|}
\hline Parameter & Values \\
\hline Number of nodes & 100 \\
\hline MAC layer & IEEE 802.11 \\
\hline Transmission range & $250 \mathrm{~m}$ \\
\hline Simulation area & $1200 \mathrm{~m} \times 1000 \mathrm{~m}$ \\
\hline Simulation time & $900 \mathrm{~s}$ \\
\hline Mobility model & Random waypoint model \\
\hline Maximum speed & $1,2,5,7,10,15$ and $20 \mathrm{~m} / \mathrm{s}$. \\
\hline Pause time & $0 \mathrm{~s}$ \\
\hline Traffic type & CBR \\
\hline Packet size & 512 bytes \\
\hline Packet rate & $2,4,8$ packets $/ \mathrm{s}$ \\
\hline Number of flows & 5 \\
\hline Number of runs per data point & 30 \\
\hline
\end{tabular}

The performance of the three techniques is measured by: throughput, end-to-end delay and routing overhead. The throughput is the amount of data received at the final destination over the simulated time averaged over number of flows. This measure provides an indication of the efficiency of the technique as it shows the amount of data that the protocol is able to deliver to destinations. End-to-end delay is the average time interval between the generation of a packet in a source node and the successful delivery of the packet at the destination node. It counts all possible delays that can occur in the source and all intermediate nodes. Routing overhead is the number of routing (control) packets sent throughout the simulated time. The smaller this value the better the performance and the more efficient the usage of resources.

Figures 3 to 5 show the throughput for the three traffic aware techniques: load density, traffic density and nodal activity. Fig. 3 demonstrates the performance of the techniques in the presence of 5 flows with traffic rate of 2 packets per second. The figure shows a decrease in throughput for all techniques as the mobility increases. This is because the increase in mobility causes frequent topology changes resulting in broken routes. Nonetheless, the figure shows that our load density achieves better throughput than the other techniques, under various mobility speeds, while traffic density shows better throughput compared to nodal activity.

Fig. 4 depicts the behaviour of the three techniques under medium traffic rate of 4 packets per second. The figure shows that load density continues to outperform both nodal activity and traffic density. Also, it shows that varying the speeds has affected the achieved throughput of all techniques but the throughput difference between the techniques is almost the same. Fig. 5 depicts the performance under the higher traffic rate of 8 packets per second. The figure shows clearly that load density exhibits better performance than both nodal activity and traffic density.

Figures 6 to 8 demonstrate the end-to-end delay for traffic density, nodal activity and load density. Fig. 6 shows clearly that load density outperforms both nodal activity and traffic density with a difference up to $300 \mathrm{~ms}$ compared to nodal activity and compared to traffic it is up to $100 \mathrm{~ms}$. Also, in Fig. 7 at a packet rate of 4 packets per second, load density exhibits better end-to-end delay performance, with a difference up to $100 \mathrm{~ms}$ compared to traffic density and compared to nodal activity it is up to $200 \mathrm{~ms}$. However when it comes to high traffic rate, as it is shown in Fig. 8, the traffic density and nodal activity techniques exhibit similar performance, where as load density still outperforms both techniques especially at high speeds.

Figures 9 to 11 show the routing overhead for the three techniques under light, moderate and high traffic with rates of 2, 4 and 8 packets per second, respectively. Fig. 9 shows that load density clearly outperforms the other techniques, where as traffic density and nodal activity techniques exhibit close performance. The difference between traffic density and nodal activity increases when increasing the traffic rate to 4 packets per second as it is shown in Fig. 10 in favour of nodal activity. However still load density has advantage over both traffic density and nodal activity. Finally, Fig. 11 demonstrates the behaviour of the three techniques under high traffic rate of 8 packets per second. The figure shows traffic density with the highest overhead and load density with the lowest overhead and hence load density is the one with better performance.

\section{Conclusions and future work}

This study has suggested a new traffic aware technique, referred here to as load density that can overcome the limitations of the existing methods in reactive routing protocols. It has also conducted a performance evaluation of the new method against the two existing similar methods, notably, degree of nodal activity and traffic density under various working environments. Simulation results have revealed that in most circumstances the load density method exhibits superior performance in terms of throughput and end-to-end delays. As a next step of this research, we plan to carry out further investigation on the performance of the techniques considering other working conditions by changing the node mobility pattern, traffic patterns, network size, and topology area. 


\section{References}

[1] D.B. Johnson, Routing in ad hoc networks of mobile hosts, Proc. Workshop on Mobile Computing Systems and Applications, IEEE Computer Society, Santa Cruz, CA, December 1994 pp. 158-163.

[2] W. Chen, N. Jain and S. Singh, ANMP: Ad hoc network management protocol, IEEE Journal on Selected Areas in Communications, Vol. 17, No. 8, August 1999, pp. 1506-1531.

[3] D.B. Johnson and D.A. Maltz, Dynamic Source Routing in ad hoc wireless networks, In Mobile Computing, edited by T. Imielinski and H. Korth, Chapter 5, Kluwer Publishing Company, 1996, pp. 153-181.

[4] Z. Haas and M. Pearlman, The performance of Query Control Schemes for the Zone Routing Protocol, IEEE/ACM Transactions on Networking, Vol. 9, Issue 4, August 2001, pp. 427-438.

[5] C.E. Perkins and P. Bhagwat, Highly dynamic DestinationSequenced Distance Vector routing (DSDV) for mobile computers, Proc. ACM SIGCOMM'94, London, September 1994, pp. 234-244.

[6] C.-C. Chiang, Routing in clustered multihop, mobile wireless networks with fading channel, Proc. of IEEE SICON'97, April 1997, pp. 197-211.

[7] S. Murthy and J.J. Garcia-Luna-Aceves, An efficient routing protocol for wireless networks, ACM Mobile Networks and Application, Special Issue on Routing in Mobile Communication Networks, Vol. 1, No. 2, October 1996, pp. 183-197.

[8] T. Clausen and P. Jacquet, Optimized Link State Routing protocol, IETF MANET, Internet Draft, Jul. 2003.

[9] R. Ogier, F. Templin and M. Lewis, Topology Dissemination Based on Reverse-Path Forwarding (TBRPF), IETF MANET, Internet Draft, Oct. 2003

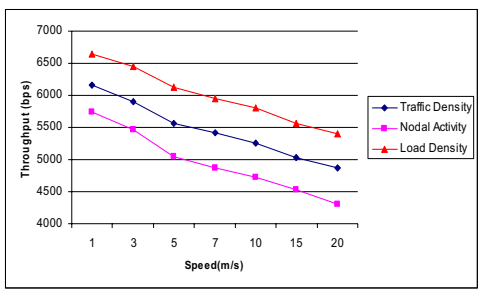

Fig. 3: Throughput under traffic with a rate of 2 packets/s.

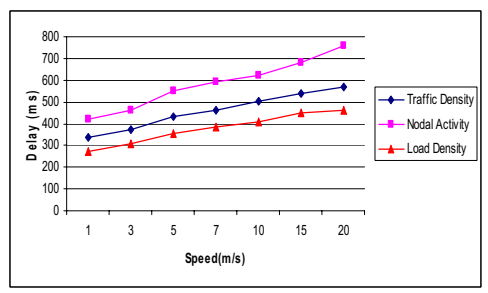

Fig. 6: Delay under traffic with a rate of 2 packets/s.

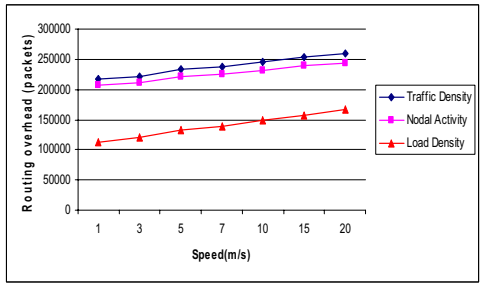

Fig. 9: Routing overhead under traffic with a rate of 2 packets/s.
[10] Y. Ko, N.H. Vaidya, Location-Aided Routing (LAR) in mobile ad hoc networks, Wireless Networks, Vol. 6, No. 4, 2000, pp. 307-321.

[11] C.-K. Toh, Associativity Based Routing for ad hoc mobile networks, Wireless Personal Communications Journal, Special Issue on Mobile Networking and Computing Systems, Vol. 4, No. 2, March 1997, pp.103-139.

[12] C.E. Perkins and E.M. Royer, Ad-hoc On-demand Distance Vector routing, Proc. 2nd IEEE Workshop on Mobile Computing Systems and Applications, New Orleans, LA, Feb. 1999, pp. 90-100.

[13] V.D. Park and M.S. Corson, A highly adaptive distributed routing algorithm for mobile wireless networks, Proc. of IEEE INFOCOM '97, Kobe, Japan, April 1997, pp. 1405-1413.

[14] M.R. Pearlman, Z.J. Haas, P. Sholander, and S.S. Tabrizi, On the impact of alternate path routing for load balancing in mobile ad hoc networks, Proc. ACM MobiHoc, 2000, pp. 3-10.

[15] V. Saigal, A.K. Nayak, S.K. Pradhan and R. Mall, Load balanced routing in mobile ad hoc networks, Computer Communications, Vol. 27, Issue 3, Feb. 2004, pp. 295-305.

[16] H. Hassanein and A. Zhou, Load-aware destination-controlled routing for MANETs, Computer Communications, Vol. 26, Issue 14, Sep. 2003, pp. 1551-1559.

[17] S.B. Lee, Jiyoung Cho, and A.T. Campbell, A hotspot mitigation protocol for ad hoc networks, Ad hoc Networks Journal, Vol. 1, No. 1, March 2003.

[18] J. Gao and L. Zhang, Load balanced short path routing in wireless networks, INFOCOM 2004, Vol. 2, March 2004, pp. 1098-1107.

[19] The Network Simulator ns-2, http://www.isi.edu/nsnam/ns.

[20] M. K. Marina and S. R. Das, On-demand multipath distance vector routing in ad hoc networks, Proc. International Conference for Network Protocols (ICNP), Nov. 2001, pp. 14-23.

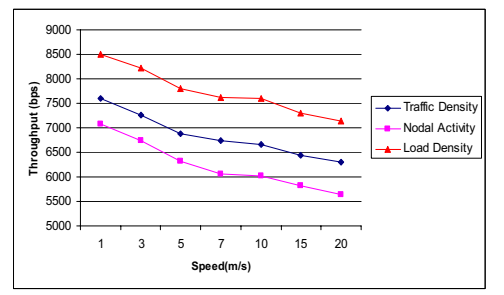

Fig. 4: Throughput under traffic with a rate of 4 packets/s.

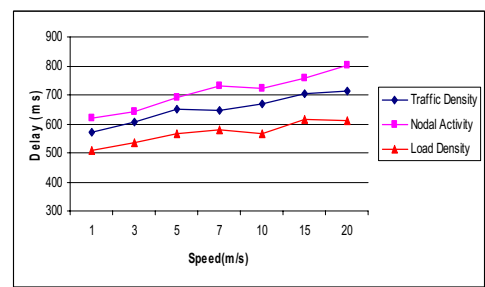

Fig. 7: Delay under traffic with a rate of 4 packets/s.

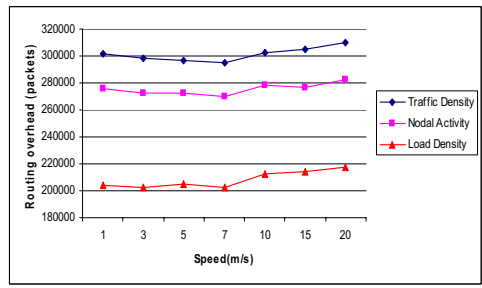

Fig. 10: Routing overhead under traffic with a rate of 4 packets/s.

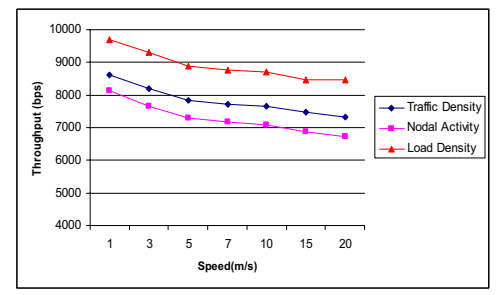

Fig. 5: Throughput under traffic with a rate of 8 packets/s.

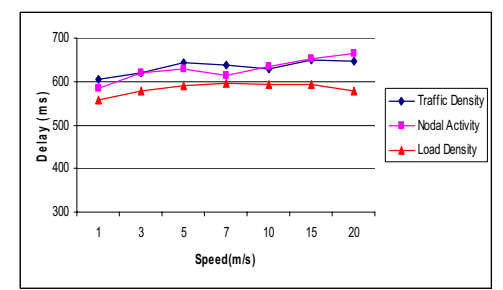

Fig. 8: Delay under traffic with a rate of 8 packets/s.

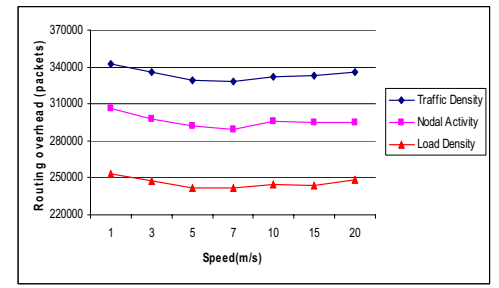

Fig. 11: Routing overhead under traffic with a rate of 8 packets/s. 